\title{
Sobrevida de pessoas vivendo com HIV/AIDS na região dos Campos Gerais, Paraná
}

\section{8-2018}

\author{
Survival of people living with HIV/AIDS in the Campos Gerais region, Paraná 2008-2018
}

Supervivencia de personas que viven con VIH/SIDA en la región Campos Gerais, Paraná 2008-2018

Recebido: 23/12/2020 | Revisado: 02/01/2021 | Aceito: 03/01/2021 | Publicado: 05/01/2021

\author{
Jean Fernando Sandeski Zuber \\ ORCID: https://orcid.org/0000-0001-5708-3300 \\ Fundação Municipal de Saúde de Ponta Grossa, Brasil \\ E-mail: jean2202@gmail.com \\ Erildo Vicente Müller \\ ORCID: https://orcid.org/0000-0003-4643-056X \\ Universidade Estadual de Ponta Grossa, Brasil \\ E-mail: erildomuller@hotmail.com \\ Pollyanna Kássia de Oliveira Borges \\ ORCID: https://orcid.org/0000-0002-9390-0459 \\ Universidade Estadual de Ponta Grossa, Brasil \\ E-mail: pkborges@uepg.br
}

\begin{abstract}
Resumo
Objetivo: Analisar o tempo de sobrevida de pessoas diagnosticadas com a infecção pelo HIV e óbitos com menção de HIV/AIDS, associados a terapia antirretroviral no período de 2008 a 2018, em residentes na região dos Campos Gerais/Paraná. Metodologia: Trata-se de um estudo de coorte retrospectivo, de caráter exploratório e documental. Resultados: estudo foi composto 737 pessoas, destas 56,9\% correspondiam a casos de óbitos e 43,1\% a pessoas vivas diagnosticadas no período de avaliação. Verificou-se que 59,3\% eram do sexo biológico masculino, 77,6\% de raça e/ou cor da pele branca e 58,6\% com ensino fundamental. A sobrevida média foi de 8,8 anos, com mulheres apresentado 10,2 anos, analfabetos com 7,1 anos e aqueles com idade superior a 60 anos de idade com 5 anos de sobrevida. Constatou-se também que as pessoas vivendo com HIV sem uso de antirretrovirais tiveram sobrevida média muito baixa, apenas 4,7 anos. Apesar das disparidades entres os subgrupos avaliados, a TARV apresenta impacto significativo no tempo de sobrevida quando o tratamento é instituído de forma precoce. Conclusão: Pesquisas de sobrevida, possibilitam monitorar os desiquilíbrios na assistência em saúde, tratamento e cuidados, situações essas que influenciam diretamente na qualidade e expectativa de vida de pessoas vivendo com HIV.
\end{abstract}

Palavras-chave: Análise de sobrevida; HIV; AIDS; Antirretrovirais.

\begin{abstract}
Objective: The aim of the study was to analyze the survival time of people diagnosed with an HIV infection and deaths with mention of HIV/AIDS, associated with antiretroviral therapy from 2008 to 2018, in residents in the region of Campos Gerais - Paraná. Methodology: It is a retrospective, exploratory and documentary cohort study. Results: The research consisted of 737 people, which $56.9 \%$ correspond to cases of death and $43.1 \%$ to living people diagnosed during the evaluation period. It was found that $59.3 \%$ were male, $77.6 \%$ of race and/or skin color white and $58.6 \%$ with elementary school. The median survival was 8.8 years, with women aged 10.2 years, illiterates aged 7.1 years and those over 60 years of age with 5 years of survival. It was also found that people living with HIV without using antiretrovirals had very low average survival, only 4.7 years. Despite the disparities between the subgroups evaluated, TARV has a significant impact on survival time when treatment is started early. Conclusion: Survival surveys make it possible to monitor imbalances in health care, treatment and care, situations that directly influence the quality and life expectancy of people living with HIV.
\end{abstract}

Keywords: Survival analyses; HIV; AIDS; Anti-retroviral agents.

\section{Resumen}

Objetivo: Analizar el tiempo de supervivencia de personas diagnosticadas con infección por VIH y muertes con mención de VIH/SIDA, asociadas a terapia antirretroviral de 2008 a 2018, en residentes de la región de Campos Gerais / Paraná. Metodología: Se trata de un estudio de cohorte retrospectivo, exploratorio y documental. Resultados: se realizó un estudio con 737 personas, de las cuales el 56,9\% correspondió a casos de muerte y el 43,1\% a vivos diagnosticados durante el período de evaluación. Se encontró que el 59,3\% eran hombres, el 77,6\% de raza y / o color de piel blanco y el 58,6\% de primaria. La mediana de supervivencia fue de 8,8 años, con mujeres de 10,2 años, analfabetas de 7,1 años y mayores de 60 años con 5 años de supervivencia. También se encontró que las personas que viven con el VIH sin usar antirretrovirales tenían una supervivencia promedio muy baja, solo 4,7 años. A pesar de las 
disparidades entre los subgrupos evaluados, el TAR tiene un impacto significativo en el tiempo de supervivencia cuando el tratamiento se inicia temprano. Conclusión: Las encuestas de supervivencia permiten monitorear los desequilibrios en la atención, el tratamiento y la atención de la salud, situaciones que influyen directamente en la calidad y esperanza de vida de las personas que viven con el VIH.

Palabras clave: Análisis de supervivencia; VIH; SIDA; Antirretrovirales.

\section{Introdução}

Poucas doenças na história da humanidade trouxeram tantos impactos sociais e para a saúde pública como a Síndrome da Imunodeficiência Adquirida (AIDS) ${ }^{1}$. No Brasil os números relativos ao enfrentamento da AIDS, mostram uma redução significativa na taxa de deteção no país, com um decréscimo de 18,7\% quando comparado o ano de 2012 (21,9/100.000 habitantes) a 2019 (17,8/100.000 habitantes), essa diminuição foi mais visível a partir de dezembro de 2013, quando ocorre a indicação para o tratamento de todas as pessoas vivendo com HIV (PVHIV), independentemente da contagem de linfócitos TCD4+ (Ministério da Saúde, 2020; Vieira et al., 2015).

A utilização precoce dos antirretrovirais no tratamento da PVHIV melhora substancialmente a qualidade de vida, reduz a transmissibilidade, diminui a incidência do HIV e dos casos de AIDS na população, além de amenizar a morbimortalidade e prolongar a sobrevida (Loch et al., 2020; Montaner et al., 2014; Pereira et al., 2018)

Com a utilização regular da terapia antirretroviral (TARV), consegue-se inibir a replicação do HIV, proporcionando níveis indetectáveis de carga viral no plasma sanguíneo evento que interrompe o avanço para a AIDS e evita a morte prematura, além de sugerir que para cada $1 \%$ de PVHIV suprimidas com TARV, há também a redução de $1 \%$ da incidência do HIV na população (Montaner et al., 2014).

O sucesso no aumento da sobrevida das PVHIV depende de fatores diretamente relacionados ao acesso às medicações, aos serviços de saúde e especialmente a adesão à terapia. Assim, a análise de sobrevivência de PVHIV pode ser considerada como uma das grandes ferramentas para se avaliar a efetividade do enfrentamento da epidemia e o impacto que as medidas públicas de saúde são capazes de modificar na história natural da doença, bem como na vida das PVHIV (Melo, Donalisio \& Cordeiro, 2017).

No Brasil as pesquisas voltadas a estudar a sobrevivência de PVHIV estão concentradas quase que majoritariamente em grandes centros urbanos e a compreensão do cenário nacional através da simples extrapolação e agregação destas informações torna-se difícil e complexa, fato que inevitavelmente poderá distorcer a compreensão sobre a problemática da sobrevida no país, pois normalmente a multiplicidade dos contextos em que a população brasileira está inserida, não é considerada (Mangal et al., 2019).

Diante disso, o objetivo deste estudo foi analisar o tempo de sobrevida de pessoas diagnosticadas com a infecção pelo HIV e óbitos com menção de HIV/AIDS, associados a utilização da terapia antirretroviral no período de 2008 a 2018, em residentes na região dos Campos Gerais no estado do Paraná.

\section{Metodologia}

Trata-se de um estudo de coorte retrospectivo, de caráter exploratório e documental de pessoas com diagnóstico para a infecção pelo HIV ou AIDS, residentes na região dos Campos Gerais/PR.

Os dados foram obtidos com base no Sistema de Informação de Agravos de Notificação (SINAN), Sistema de Controle de Exames Laboratoriais da Rede Nacional de Contagem de Linfócitos (SISCEL), Sistema de Controle Logístico de Medicamentos (SICLOM), Sistema de Informação sobre Mortalidade (SIM), Serviço Funerário Municipal de Ponta Grossa e por registros nos prontuários físicos no ambulatório do Serviço de Assistência Especializada (SAE) do município de Ponta Grossa/Pr. Este serviço além de ser responsável pelos atendimentos de infectologia da cidade onde está inserido, é também 
referência para os outros 11 municípios que compõem a $3^{\mathrm{a}}$ Regional de Saúde do Estado do Paraná ( $\left.3^{\mathrm{a} R S}\right)$, no que se refere a assistência a PVHIV e pessoas com diagnósticos de hepatites virais, hanseníase e casos especiais de tuberculose.

A coorte de estudo foi composta por pessoas de ambos os sexos, residentes na $3^{\mathrm{a}} \mathrm{RS}$ e com diagnóstico para HIV/AIDS no período de 2008 a 2018 e todos os óbitos para os mesmos diagnósticos ocorridos no neste intervalo de tempo.

Preliminarmente, foram compostos dois grupos, sendo denominados "Grupo 1 - Caso Vivo" e Grupo 2 - Óbitos. O primeiro foi formado a partir das notificações compulsórias para HIV e AIDS registradas no período de 2008 a 2018, excluídos os casos de transmissões verticais, gestantes HIV e com encerramento "óbito", não havendo retorno para casos de transmissão sexual ou pelo uso de drogas injetáveis em crianças. Como resultado obteve-se 1214 pessoas vivas notificadas para HIV/AIDS, porém com 46 casos duplicados, sendo estes excluídos, o que caracterizou 1168 notificações elegíveis para o cálculo amostral. O cálculo para o tamanho da amostra, foi realizada pelo método de amostragem aleatória simples com 95\% de confiança e 5\% de precisão para um total de 1168 pacientes vivos, o que determinou o estudo de 289 casos, acrescido de $10 \%$ como índice de segurança técnica, totalizando uma amostra final $\mathrm{n}=318$ pacientes vivos a serem estudados.

O segundo grupo foi composto por todos os casos pessoas diagnosticadas com HIV/AIDS independente do ano, mas com encerramento de suas notificações compulsórias por motivo óbito durante o período de pesquisa, obedecido o critério de residência. A busca retornou 419 casos de óbitos a serem estudados.

Para verificar o acompanhamento dos usuários no SAE, foi considerado o número de dispensações/retiradas da TARV na farmácia especializada do SAE, sendo essas baseadas em 30 dias de uso. Este levantamento ocorreu mensalmente durante o todo o período de pesquisa e para todas as pessoas incluídas neste estudo. A partir destas informações foi possível mensurar a assiduidade das PVHIV ao tratamento, trata-se de uma variável em percentual e corresponde a porcentagem de assiduidade que paciente apresentou durante o tratamento no período do estudo. Esta variável foi construída utilizando razão entre a variável número de vezes que o paciente retirou medicação e número máximo de vezes que o paciente poderia retirar medicação.

A análise descritiva dos dados ocorreu com a estimativa de média, mediana, desvio padrão, percentil $25 \%$ e $75 \%$ das variáveis quantitativas e frequências simples e relativas das variáveis qualitativas. Em seguida, verificou-se a aderência das quantitativas quanto à distribuição normal pelo teste Shapiro-Wilk, verificou-se que as variáveis de interesse não apresentaram distribuição normal. Portanto, a diferença entre os pacientes em óbito e vivos foi avaliada com o teste U de Mann-Withney. A sobrevida foi avaliada com curvas de Kaplan-Meier e foram realizadas comparações entre sobrevidas entre grupos com o teste de Breslow. Os testes foram considerados significativos quando $\mathrm{p}<0,05$ e as análises foram realizadas no SPSS 21.0 (IBM, 2012).

Dentre as variáveis pesquisas neste estudo, optou-se também em tentar observar a relação da sobrevida com as primeiras mudanças ocorridas a partir da indicação precoce para o tratamento do HIV, o qual estava baseado em uma nova política pública de tratamento para todos. No Brasil, a partir do mês de dezembro de 2013, o Ministério da Saúde através do Protocolo Clínico de Diretrizes Terapêuticas para HIV (PCDT-HIV) passou a orientar o início da terapia com antirretrovirais para todas as PVHIV, permitindo que o tratamento seja indicado imediatamente após a confirmação do diagnóstico da infecção pelo HIV, independentemente da situação imunológica, baseada na contagem de LT-CD4 (Brasil, 2013; Brasil, 2018; Ministério da Saúde, 2020; World Health Organization, 2016).

Para avaliar esta hipótese, foi realizado um recorte dos pacientes para pareamento. Dado que no universo do estudo $(\mathrm{n}=737)$, foi composto por diagnósticos anteriores e posteriores a 2008. Assim, tempo máximo de sobrevida das pessoas diagnosticadas até 2013 era superior ao tempo máximo de sobrevida daquelas com diagnóstico depois de 2013. Então, fez-se necessária uma seleção como forma de pareamento do acompanhamento máximo.

Para o grupo óbito, foram selecionadas pessoas com diagnóstico e óbito entre jan/2008 e jun/2013 para compor o grupo pré-tratamento universal e com diagnóstico e óbito entre jul/2013 e dez/2018 para compor o grupo pós-tratamento 
universal. Para o grupo vivo, foram selecionadas pessoas com diagnóstico entre jan/2008 e jun/2013, considerando como follow-up até jun/2013 para compor o grupo pré-tratamento universal e pacientes com diagnóstico entre jul/2013 e dez/2018, considerando follow-up até dez/2018 para compor o grupo pós-tratamento universal.

Desta forma, no grupo pré e pós-tratamento universal, o tempo máximo de acompanhamento foi de 5,5 anos com pacientes vivos e em óbito, independentemente do tempo que contribuiu na série histórica completa. Este recorte gerou um $\mathrm{n}=$ 487 pacientes, sendo 205 em óbito (92 pré e 113 pós) e 282 vivos (115 pré e 167 pós).

Esta pesquisa integra um projeto principal maior, aprovado pelo comitê de ética em pesquisa, sob o parecer número: 2.631.445 e certificado de apresentação para apreciação ética n 87864518.0.0000.0105 na data de 02/05/2018, com o título principal “Contextos epidemiológicos, biológicos e de qualidade de vida de indivíduos convivendo com HIV/AIDS”.

\section{Resultados}

O estudo foi composto 737 pessoas diagnosticadas com HIV/AIDS. Deste quantitativo 56,9\% (n=419) foram casos de óbitos ocorridos durante o período de avaliação, os outros 43,1\% compreendem as pessoas vivas diagnosticadas entre 2008 a 2018. Verificou-se que as PVHIV eram predominantemente compostas pelo sexo biológico masculino com 59,3\% ( $\mathrm{n}=437$ ). A raça e/ou cor da pele 77,6\% ( $\mathrm{n}=560)$ eram brancos; pretos, pardos, amarelos e indígenas somaram 18,2\% ( $\mathrm{n=134)}(\mathrm{Tabela} 1)$.

Evidencia-se também que 65,4\% (n=432) dos diagnósticos para HIV/AIDS ocorreram até 31/12/2013 e os outros $34,6 \%(n=225)$ a partir desta data até o fechamento do estudo.

Tabela 1 - Caracterização da população estudada, segundo óbito, sexo, raça/cor, escolaridade e ano do diagnóstico, assiduidade, SAE, Ponta Grossa Paraná, 2008-2018.

\begin{tabular}{llrr}
\hline $\mathbf{N = 7 3 7}$ & & $\mathbf{N}$ & $\mathbf{\%}$ \\
\hline \multirow{2}{*}{ Óbito } & Não & 318 & $43,1 \%$ \\
& Sim & 419 & $56,9 \%$ \\
\hline \multirow{3}{*}{ Sexo } & Feminino & 298 & $40,4 \%$ \\
& Masculino & 437 & $59,3 \%$ \\
& Não informado & 2 & $0,3 \%$ \\
\hline \multirow{3}{*}{ Raça/Cor da pele } & Branca & 560 & $77,6 \%$ \\
& Não branca & 134 & $18,6 \%$ \\
& Ignorado/não informado & 28 & $3,9 \%$ \\
\hline \multirow{4}{*}{ Escolaridade } & Analfabeto & 14 & $1,9 \%$ \\
& Ensino fundamental (completo/incompleto) & 432 & $58,6 \%$ \\
& Ensino médio (completo/incompleto) & 157 & $21,3 \%$ \\
& Ensino superior (completo/incompleto) & 50 & $6,8 \%$ \\
\multirow{2}{*}{ Diagnóstico } & Ignorado/não informado & 84 & $11,4 \%$ \\
\hline & Até 12/2013 & 482 & $65,4 \%$ \\
& 01/2014 a 12/2018 & 255 & $34,6 \%$ \\
\hline \multirow{3}{*}{ Assiduidade ao tratamento } & 161 & $21,8 \%$ \\
& $0 \%$ & 67 & $9,1 \%$ \\
& 1 a 25\% & 135 & $18,3 \%$ \\
& 26 a 50\% & 167 & $22,7 \%$ \\
& 51 a 75\% & 187 & $25,4 \%$ \\
& 76 a 100\% & 20 & $2,7 \%$ \\
\hline
\end{tabular}

Fonte: Autores. 
Com relação à assiduidade ao tratamento percebe-se que $25 \%(\mathrm{n}=187)$ das PVHIV estudadas tiveram frequência de tratamento de $76 \%$ a $100 \%$, enquanto $21,8 \%(\mathrm{n}=161)$ nunca foram acompanhados pelo serviço de saúde especializado (Tabela 01), sendo a média geral este subgrupo de apenas 49,1\% (Tabela 2).

A média de idade geral das PVHIV no estudo foi 37,5 anos (dp=12,1), com amplitude 1 até 74,4 anos, e idade média ao final do acompanhamento de 42,9 anos (dp=11,8), sendo a menor de 0,6 e a maior 79,2 anos (Tabela 02). Em alusão ao tempo de acompanhamento no estudo, verifica-se que a média para a população foi 5,4 anos (dp=5,3), com o equivalente a 1973,4 dias (dp=1920), sendo a assiduidade global avaliada com uma média de 49,1\% ( $\mathrm{dp}=36,0 \%)$ (Tabela 2).

Tabela 2 - Análise estatística inferencial dos grupos de casos vivos e óbitos, segundo idade ao diagnóstico, idade ao final no acompanhamento, tempo de acompanhamento em anos, meses e dias, assiduidade, SAE, Ponta Grossa, Paraná, $2008-2018$.

\begin{tabular}{|c|c|c|c|c|c|c|c|c|}
\hline $\mathrm{n}=\mathbf{7 3 7}$ & $\mathbf{M}$ & DP & Mín & Máx & MD & $\begin{array}{l}\text { Perc } \\
25 \%\end{array}$ & $\begin{array}{l}\text { Perc } \\
75 \%\end{array}$ & $\begin{array}{c}\text { p-valor do } \\
\text { teste de } \\
\text { normalidade* }\end{array}$ \\
\hline Idade ao diagnóstico (anos) & 37,5 & 12,1 & 0,0 & 74,4 & 36,7 & 28,3 & 46,0 & $<0,001$ \\
\hline $\begin{array}{l}\text { Idade ao final do acompanhamento } \\
\text { (anos) }\end{array}$ & 42,9 & 11,8 & 0,6 & 79,2 & 41,8 & 34,5 & 51,4 & 0,003 \\
\hline Tempo de acompanhamento (anos) & 5,4 & $J, J$ & 0,0 & 22,9 & 4,2 & 0,4 & 8 & $<0,001$ \\
\hline Tempo de acompanhamento (meses) & 64,9 & 63,1 & 0,0 & 274,2 & 50,6 & 5,0 & 104,9 & $<0,001$ \\
\hline Tempo de acompanhamento (dias) & 1973,4 & 1920,0 & 0,0 & 8341,0 & 1539,0 & 152,0 & 3192,0 & $<0,001$ \\
\hline Assiduidade & $49,1 \%$ & $36,0 \%$ & $0,0 \%$ & $\begin{array}{c}100,0 \\
\%\end{array}$ & $50,0 \%$ & $\begin{array}{c}10,0 \\
\%\end{array}$ & $77,8 \%$ & $<0,001$ \\
\hline
\end{tabular}

M=média; DP=desvio padrão; Mín=mínimo; Máx-máximo; MD=mediana; Perc=percentil; *teste Shapiro-Wilk Fonte: Autores.

Para a idade no diagnóstico observa-se (Tabela 3) que a mediana para o grupo de óbitos foi de 38,9 anos, enquanto para o de pessoas vivas de 33,9 anos (p<0,001), e a média de tempo decorrido do diagnóstico até o final do acompanhamento foi de 2,3 anos para o primeiro e 5,3 anos no segundo ( $\mathrm{p}<0,001)$.

Tabela 3 - Grupos de casos de óbitos e vivos, segundo idade ao diagnóstico, idade ao final do acompanhamento, tempo entre o diagnóstico e acompanhamento, assiduidade, SAE, Ponta Grossa, Paraná, 2008-2018.

\begin{tabular}{|c|c|c|c|c|c|c|c|}
\hline \multirow[b]{2}{*}{$\mathbf{n}=\mathbf{7 3 7}$} & \multirow[b]{2}{*}{ MD } & \multicolumn{5}{|c|}{ Óbito } & \multirow[b]{2}{*}{$\begin{array}{c}\text { p- } \\
\text { valor* }\end{array}$} \\
\hline & & $\begin{array}{l}\text { Sim } \\
\text { Perc } \\
25 \%\end{array}$ & $\begin{array}{l}\text { Perc } \\
75 \% \\
\end{array}$ & MD & $\begin{array}{l}\text { Não } \\
\text { Perc } \\
25 \% \\
\end{array}$ & $\begin{array}{l}\text { Perc } \\
75 \% \\
\end{array}$ & \\
\hline Idade ao diagnóstico (anos) & 38,9 & 30,0 & 47,4 & 33,9 & 25,8 & 44,9 & $<0,001$ \\
\hline Idade ao final do acompanhamento (anos) & 42,9 & 35,6 & 52,1 & 40,6 & 32,5 & 50,3 & 0,004 \\
\hline $\begin{array}{l}\text { Tempo entre diagnóstico e final do acompanhamento } \\
\text { (anos) }\end{array}$ & 2,3 & 0,1 & 8,6 & 5,3 & 2,7 & 8,9 & $<0,001$ \\
\hline Assiduidade & $42,9 \%$ & $0,0 \%$ & $100,0 \%$ & $57,1 \%$ & $40,0 \%$ & $70,0 \%$ & 0,043 \\
\hline
\end{tabular}

*p-valor ao teste U de Mann-Whitney de comparação entre óbito sim e não

Fonte: Autores.

A média de sobrevida da coorte foi de 8,8 anos (IC 95\% 8,2-9,5 anos). A sobrevida de mulheres e homens foi de 10,2 anos e 7,9 anos respectivamente, verificando-se diferença de sobrevida de 2,3 anos ( $\mathrm{p}=0,005)$. Verificou-se em analfabetos a sobrevida de 7,1 anos (IC 95\% 3,3-10,9 anos) e pessoas com ensino superior de 10,8 anos (IC 95\% 7,8-13,8 anos) (p=0,109) (Tabela 4).

Para o fator sobrevida relacionado à idade ao diagnóstico, evidenciou-se que a faixa-etária dos 11 aos 20 anos apresentou sobrevida de 14,3 anos, enquanto as faixas-etárias de 40-60 e acima de 60 anos, registraram 6,2 anos e 5 anos, respectivamente. 
Verifica-se ainda na tabela 4 que pessoas que não apresentaram frequência no acompanhamento ambulatorial tiveram sobrevida média de apenas de 4,7 anos, enquanto nas assiduidades intermediárias (1\% a $75 \%$ frequência) a média foi de 12,0 a 13,1 anos $(p<0,001)$, para as pessoas com frequência acima de $75 \%$ o registro foi de 4,9 anos.

A média de sobrevida acumulada para as pessoas diagnosticadas e acompanhadas até junho de 2013 foi de 3,1 anos (IC95\% 2,7-3,4) enquanto para o período de julho de 2013 a dezembro de 2018 a média foi de 3,4 anos (IC95\% 3,0-3,6) e a sobrevida acumulativa máxima para ambos os grupos de 5,5 anos (Tabela 5).

Tabela 4 - Sobrevida da coorte, segundo sexo, raça/cor da pele, escolaridade, faixa-etária ao diagnóstico, assiduidade, SAE, Ponta Grossa, Paraná, 2008-2018.

\begin{tabular}{|c|c|c|c|c|c|c|c|c|c|c|}
\hline \multirow{3}{*}{\multicolumn{2}{|c|}{$\begin{array}{l}\text { Sobrevida considerando todos os } \\
\text { pacientes }(n=737)\end{array}$}} & \multicolumn{4}{|c|}{ Média } & \multicolumn{4}{|c|}{ Mediana } & \multirow{3}{*}{$\begin{array}{l}\text { p-valor } \\
\text { entre } \\
\text { grupos* }\end{array}$} \\
\hline & & \multirow{2}{*}{$\mathbf{E}$} & \multirow{2}{*}{$\mathbf{E P}$} & \multicolumn{2}{|c|}{ IC $95 \%$} & \multirow{2}{*}{$\mathbf{E}$} & \multirow{2}{*}{ EP } & \multicolumn{2}{|c|}{ IC $95 \%$} & \\
\hline & & & & Inf & Sup & & & Inf & Sup & \\
\hline Geral & & 8,8 & 0,3 & 8,2 & 9,5 & 8,6 & 0,5 & 7,5 & 9,7 & 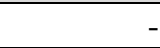 \\
\hline \multirow[t]{2}{*}{ Sexo } & Sexo feminino** & 10,2 & 0,5 & 9,2 & 11,3 & 10,2 & 1,1 & 8,1 & 12,3 & 0,005 \\
\hline & Sexo masculino & 7,9 & 0,4 & 7,1 & 8,7 & 7,3 & 0,8 & 5,8 & 8,8 & \\
\hline \multirow{2}{*}{$\begin{array}{l}\text { Raça/Cor da } \\
\text { pele }\end{array}$} & Branca & 9,3 & 0,4 & 8,5 & 10,0 & 9,4 & 0,6 & 8,2 & 10,6 & 0,411 \\
\hline & Não branca & 8,0 & 0,7 & 6,7 & 9,4 & 7,6 & 1,0 & 5,6 & 9,6 & \\
\hline \multirow[t]{4}{*}{ Escolaridade } & Analfabeto & 7,1 & 1,9 & 3,3 & 10,9 & 4,3 & 3,4 & 0,0 & 10,8 & 0,109 \\
\hline & Ensino fundamental & 8,8 & 0,4 & 7,9 & 9,6 & 8,4 & 0,7 & 7,1 & 9,7 & \\
\hline & Ensino médio & 10,2 & 0,7 & 8,8 & 11,7 & 11,5 & 0,8 & 9,8 & 13,1 & \\
\hline & Ensino superior & 10,8 & 1,5 & 7,8 & 13,8 & 10,2 & 0,7 & 8,8 & 11,5 & \\
\hline \multirow{5}{*}{$\begin{array}{l}\text { Faixa etária ao } \\
\text { diagnóstico }\end{array}$} & Até 10 anos & 8,2 & 3,7 & 1,9 & 16,4 & 6,6 & 6,3 & 0,0 & 19,0 & $<0,001$ \\
\hline & 11 a 20 anos $* *$ & 14,3 & 1,4 & 11,6 & 16,9 & 16,7 & 1,6 & 13,5 & 19,8 & \\
\hline & 21 a 40 anos & 10,0 & 0,4 & 9,2 & 10,8 & 10,0 & 0,5 & 8,9 & 11,0 & \\
\hline & 41 a 60 anos & 6,2 & 0,5 & 5,2 & 7,2 & 3,9 & 1,0 & 1,0 & 5,9 & \\
\hline & Acima de 60 anos & 5,0 & 1,2 & 2,6 & 7,4 & 4,3 & 1,1 & 1,1 & 6,5 & \\
\hline \multirow{5}{*}{$\begin{array}{l}\text { Assiduidade ao } \\
\text { tratamento }\end{array}$} & $0 \%$ & 4,7 & 0,6 & 3,6 & 5,9 & 0,1 & 0,1 & 0,0 & 0,2 & $<0,001$ \\
\hline & 1 a $25 \% * *$ & 12,0 & 0,8 & 10,4 & 13,6 & 10,8 & 10,8 & 9,4 & 12,2 & \\
\hline & 26 a $50 \% * *$ & 12,5 & 0,7 & 11,1 & 13,9 & 12,6 & 12,6 & 10,6 & 14,6 & \\
\hline & 51 a $75 \%$ & 13,1 & 0,7 & 11,7 & 14,4 & 12,8 & 12,8 & 6,8 & 18,7 & \\
\hline & 76 a $100 \%$ & 4,9 & 0,5 & 3,9 & 5,9 & 2,3 & 2,3 & 1,3 & 3,3 & \\
\hline
\end{tabular}

$\mathrm{E}=$ =stimado; $\mathrm{EP}=$ =rro padrão; $\mathrm{IC}=$ intervalo de confiança; $\mathrm{Inf}=$ inferior; Sup=superior; *p-valor ao teste de Breslow de comparação entre grupos de fatores; **significativamente maior

Fonte: Autores.

Tabela 5 - Sobrevida cumulativa dos grupos casos vivos e óbitos, segundo o período de tratamento, SAE, Ponta Grossa Paraná, 2008-2018.

\begin{tabular}{lcccc}
\hline $\begin{array}{c}\text { Sobrevida considerando recorte pré e pós- } \\
\text { tratamento universal (n=487) }\end{array}$ & Média & $\begin{array}{c}\text { Erro } \\
\text { padrão }\end{array}$ & $\begin{array}{c}\text { IC 95\% } \\
\text { Inferior }\end{array}$ & $\begin{array}{c}\text { Superior } \\
\text { grupos* }\end{array}$ \\
\hline $\begin{array}{l}\text { Diagnóstico e acompanhamento entre jan/2008 } \\
\text { e jun/2013 }\end{array}$ & 3,1 & 0,2 & 2,7 & 3,4 \\
$\begin{array}{l}\text { Diagnóstico e acompanhamento entre jul/2013 } \\
\text { e dez/2018 }\end{array}$ & 3,4 & 0,1 & 3,0 & 3,6 \\
\hline
\end{tabular}

$\mathrm{IC}=$ intervalo de confiança; *p-valor ao teste de Breslow de comparação entre grupos

Fonte: Autores. 


\section{Discussão}

Para melhor compreensão da análise dos dados, a discussão foi dividida em duas etapas. A primeira volta-se discutir a relação da infecção pelo HIV com as variáveis epidemiológicas, e a segunda parte versou para a discussão da sobrevida considerando as variáveis de interesse do estudo.

Foi observado que as pessoas vivendo com HIV/AIDS eram hegemonicamente compostas por pessoas de sexo biológico masculino, cor branca, idade média 37 anos, escolarização de até 8 anos (ensino fundamental), idade média ao diagnóstico de 33,9 anos para participantes vivos e de 38,9 anos para os casos de óbito.

Knauth e colaboradores (2020), enfatizam que os homens configuram o público mais exposto ao HIV, ao longo da história da infecção no Brasil. Perfil que também foi encontrado por outros autores, os quais também descrevem que mais da metade dos homens vivendo com HIV tinham até 8 anos de escolarização (Alexander, 2020; Figueirêdo Júnior et al., 2020; Knauth et al., 2020; Mocellin et al., 2020; Velame et al., 2020).

No perfil encontrado neste estudo, verificou-se também o predomínio de pessoas de cor branca $(75,9 \%)$, essa desproporção pode ser explicada pela própria composição populacional do estado, onde esta pesquisa foi realizada. Quando se analisa os dados nacionais para as novas infecções pelo HIV, estratificado por raça/cor da pele e sexo, os números tornam inversamente proporcionais, pois no sexo masculino as novas infecções correspondem a 48,1\% em não brancos (pretos, 9,6\% e pardos, 38,4\%) e 42,6\% em brancos; já no sexo feminino não brancas representam 53,6\% (pretas, 12,9\% e pardas, 40,7\%) das novas infecções, enquanto brancas 37,2\% (Ministério da Saúde, 2020).

Dentre as hipóteses para explicar a ausência de pessoas pretas, pardas ou mesmo indígenas, podem estar relacionadas a baixa capilarização do teste para HIV, especificamente nas comunidades mais vulneráveis, ou seja, ausência de testagem extramuros, pois normalmente a disponibilidade destes insumos está restrita as unidades de saúde; o medo da exposição sorológica nas cidades pequenas e/ou comunidades; dificuldades econômicas para o deslocamento até o serviço de referência, seja para testagem rápida ou tratamento.

Observa-se também neste estudo, o grande número de pessoas com baixa escolaridade, 58,6\% tinham até 8 anos de estudo regular, evento que somado aos analfabetos mostra frequência de $60,5 \%$, dados que também são corroborados por outros autores (Kanauth et al., 2020; Lima et al., 2018; Müller \& Borges, 2020). No contexto do HIV/AIDS a escolaridade é reconhecida como um importante indicador de influência na saúde da PVHIV (Cerqueira \& Rodrigues, 2016; Chaves, 2018). Müller \& Borges (2020), reforçam esse determinante, ao afirmarem que o nível de escolaridade pode estar relacionado as condições de vida e renda, desta forma a prevenção do HIV/AIDS pode não estar ao alcance destas pessoas menos favorecidas, ou se chegam, acabam por não ser representativas em suas histórias de vida.

Outro elemento importante está relacionado a idade no diagnóstico da infecção pelo HIV, neste estudo a média de registros foi para a terceira década de vida, mais precisamente aos 33,9 anos para aqueles permaneceram vivos até o final do estudo e de 38,9 anos para os casos identificados como óbitos. Haddad et al.(2018), corroboram esses achados, ao também identificarem a faixa-etária dos 30 aos 39 anos com maior número e proporções de notificações para HIV. Ressalta-se que no Brasil a concentração dos casos de HIV estão mais presentes na faixa etária dos 20-34 anos, enquanto os casos de AIDS na faixa-etária dos 25-39 anos (Ministério da Saúde, 2020).

Os dados observados sobre a assiduidade no tratamento demonstram quão é difícil vincular e reter a PVHIV no SAE. Vários fatores podem ser os influenciadores para este perfil contraproducente, perpassando desde o enfrentamento negativo do diagnóstico; estigma sustentado pela doença; medo quanto ao preconceito; discriminação e/ou julgamento; traumas psicológicos, onde alguns estão relacionados a violência doméstica e/ou sexual; idade (adolescentes e idosos); uso de substâncias psicoativas; "grande" período assintomático, com a falsa sensação de que o tratamento é desnecessário e pôr fim a baixa autocompreensão do seu real estado de saúde (Costa et al., 2020; Oliveira et al., 2015). 
Entendendo que a adesão terapêutica é complexa, dinâmica e multifatorial, outros estudos que avaliaram essa questão, como de Betancur et al. (2017), Miyada et al. (2017), e Oliveira et al. (2015) também identificaram níveis baixos e/ou insatisfatórios de adesão em 51\%, 80\%, e 78,8\% entre as PVHIV em TARV, respectivamente.

Uma das hipóteses para explicar a baixa adesão entre os usuários do SAE, pode estar relacionada a forma de atendimento realizado, pois observou-se que até 2014, o registro das informações no prontuário dos pacientes era quase que exclusivamente realizado pelo médico, evento que descartaria uma assistência interdisciplinar. Essa situação pode ter centrado grande parte dos atendimentos somente na prescrição TARV e profilaxia para infecções oportunistas, deixando de se observar a integralidade e a complexidade envolvida no atendimento a PVHIV, prejudicando assim o entendimento sobre a doença e a importância do tratamento.

Neste estudo observou-se que idade no diagnóstico foi associada a um efeito protetor em relação ao grupo composto pelos casos de óbito, a menor mediana para a idade na descoberta do HIV, esteve também relacionada a uma maior evidencia de assiduidade no tratamento. Estes fatores foram similares aos encontrados no estudo realizado no Irã para o qual a média de idade foi 33,2 anos (dp=9,6), os autores observaram que a idade possuía influência positiva na taxa de sobrevivência, sugerindo ainda que a TARV reduziu a progressão do HIV e a morte (Akbari et al., 2019).

Neste sentido, a adesão terapêutica tem impacto direto e significativo para a sobrevida da PVHIV, reconhece-se ainda que a TARV reduz significativamente a mortalidade e morbidade destas pessoas, sugerindo que a sobrevida é comparável com a população em geral (May et al., 2014). Contudo, ainda não há certeza se existe uma lacuna para a sobrevida de PVHIV com acesso a assistência em saúde quando comparado a população em geral (Trickey et al., 2017). Alguns autores tem mostrado discrepâncias quanto as taxas de mortalidade em PVHIV (May et al., 2014). De Coninck et al.(2018) em pesquisa com 12.138 (4066 PVHIV e 8072 controles HIV-não reagentes) no período de 1996 a 2011 descreve que apesar do tratamento realizado com sucesso e com supressão viral, as PVHIV foram 3 vezes mais propensas a morrer quando comparado ao grupo controle.

Mediante o exposto a análise sobrevivência neste estudo, identificou sobrevida média de 8,8 anos, com maior sobrevida entre mulheres. Dados semelhantes foram descritos por Zhang et al.(2016) em uma coorte realizada na cidade Liangshan, sudoeste da China (período 2005-2003), os autores mostraram sobrevida global para pacientes em uso TARV de 94,3 meses (7,8 anos), sendo para o sexo feminino de 100,4 meses (8,3 anos) e sexo masculino 91,5 meses (7,6 anos). Uma margem bem inferior de sobrevida pode ser vista na pesquisa de Melo et al.(2019) realizada no Brasil, para a qual em mulheres observou-se 75,1 meses (6,2 anos) de sobrevida, enquanto para homens 65,7 meses (5,4 anos), no período de 1998 a 2009.

No Brasil as políticas públicas para a saúde da mulher estão bem consolidadas, apresentando reflexo direto na redução da morbimortalidade feminina. Sabe-se que mulheres são estimuladas desde muito cedo a frequentar os serviços de saúde e esse comportamento também é visualizado na prevenção e tratamento para HIV/AIDS, pois mulheres em virtude do pré-natal são oportunizadas com no mínimo três testagens rápidas para HIV durante o período gestacional e parto (Brasil, 2019). Para aquelas que vivem com HIV, somam-se ainda cuidados intensivos desprendidos pelas equipes de saúde a fim de se evitar uma possível transmissão vertical. Situações como essas, colocam as mulheres em evidencia assistencial, sendo muito mais propensas a buscas ativas, monitoramento e acompanhamento (Brasil, 2018; FIALHO et al., 2020)

Embora em períodos e tempos (número de anos) distintos de avaliação, outros autores chegaram a resultados similares aos encontrados nesta coorte em relação a sobrevida nas PVHIV, Zeng et al. (2019) obteve 11,5 anos sobrevivência (período 1991-2017) na pesquisa com PVHIV geral; Glynn et al. (2007) encontrou 10,5 anos (período 1991-2002) na pesquisa com mineiros; Lavreys et al.(2006) por sua vez evidenciou uma sobrevida de 8,7 anos (período 1993-2004) em estudo com profissionais do sexo; Morgan et al. (2002) observou 9,8 anos de sobrevida (período de 1990-2000) no estudo com moradores zona rural. 
Com a oferta e evolução dos ARVs utilizados no tratamento da AIDS, verifica-se que o curso da doença começou a ser modificada em todos os contextos, sobretudo com resultado positivo para a sobrevida, pois no início da pandemia pouco se sabia sobre a AIDS e sua gravidade (Oliveira et al., 2015). Rossi et al. (2012) menciona que a média de sobrevida era extremamente baixa no período de 1986-1997, sendo de apenas 10,3 meses. Por sua vez, Pereira et al. (2018), identificaram um cenário mais sombrio, mostrando sobrevivência média para os anos de 1982-1989 de apenas 5,1 meses, com uma melhora substancial para 16 meses, em 1995 e 58 meses em 1996.

Diante da oferta do tratamento para a AIDS no Brasil, Tancredi \& Waldman (2014), relatam o impacto dos ARVs, ao estudar uma coorte de 6.594 pessoas diagnosticadas com AIDS na cidade de São Paulo, em períodos distintos, entre 1988-1993 a sobrevida encontrada foi de 13,4 meses com 1.552 mortes; para 1994-1996 a sobrevivência alcançou a média de 22,3 meses com 731 mortes; e na era HAART de 1997-2003 a sobrevida acumulativa foi de 72\% para o acompanhamento máximo de 108 meses.

No presente estudo foi observado ainda, que a idade ao diagnóstico é um importante preditor de sobrevida, pois pessoas diagnosticadas com HIV/AIDS nas faixas etárias de menor idade, tiveram sobrevida média significativamente maior do que aqueles com idades superiores. Por outro lado, as PVHIV nas faixas etárias de 41 a 60 anos e superior a 60 anos no diagnóstico, apresentaram o fator idade como um indicador extremamente negativo, com média de sobrevidas muito baixas de apenas 6,2 e 5 anos, e com medianas ainda menores de 3,9 e 4,3 anos, respectivamente.

Corroborando os dados do presente estudo, Glynn et al. (2007), observaram média de sobrevida de 11,5 anos na faixaetária dos 15-24 anos; 10,5 anos entre 25 a 34 anos; 9,5 anos para pessoas entre 35 a 44 anos e 6,3 anos para aqueles com mais de 45 anos. Na revisão realizada por Jaffar et al. (2004) com estudos para populações africanas o tempo médio de sobrevida foi de 12,5 anos, 10,9 anos, 9,1 anos paras as respectivas faixas-etárias 15-24 anos, 25-34 anos e 35-44 anos; para aqueles com idade entre 45-54 anos a sobrevida média foi de 7,9 anos.

Esta pesquisa também se propôs a verificar a sobrevida em relação a raça/cor da pele e nível de escolaridade, porém nas análises, essas variáveis não se mostraram estatisticamente significativas quando comparadas aos respectivos subgrupos, registrando amplitude média de 7,1 a 10,8 anos.

Entretanto, há fortes indícios de que as disparidades na sobrevida de pretos e brancos, Earnshaw et al. (2015) destacam que o HIV é uma epidemia alimentada, em parte, pelo estigma estrutural, e a análise da interseção raça e gênero são fundamentais, pois podem afetar diretamente a sobrevida de pessoas negras/pretas vivendo com HIV, pois esse seguimento populacional apresenta taxas mais elevadas para a mortalidade e ainda são menos propensos a permanecer vivos após 9 anos do diagnóstico do HIV, quando comparados a brancos, latinos e asiáticos.

Quanto a sobrevida relacionada a escolaridade, Cobre et al., (2020) afirmam que pacientes que concluíram o ensino fundamental apresentaram menor probabilidade de sobrevivência quando comparados àqueles com ensino médio ou técnico/profissional. Contudo, Palumbo (2015) faz uma indagação interessante ao sugerir PVHIV com conhecimento sobre as condições de saúde parecem compreender melhor a relação saúde-doença, porém as com pouca alfabetização em saúde, apresentam maior conformidade com as orientações e prescrições profissionais, confiando cegamente nestes.

Neste sentido, uma das hipóteses para que a sobrevida de pessoas analfabetas e com graus intermediários de escolaridade se assemelhassem aos com maior instrução neste estudo, esteja ligada a resignação para o tratamento, através da imposição da terapia, ou seja, no modo imperativo com que os tratamentos são manejados pelos profissionais de saúde, condição a qual pode apresentar duas situações distintas, sendo a primeira com a aceitação incondicional da terapia, ou a segunda com o não entendimento do porquê o tratamento é necessário, fato que culminaria com a má adesão.

Outro dado evidenciado nesta pesquisa, está relacionado a diferença significativa na sobrevida quando analisada sob perspectiva da assiduidade (frequência) no tratamento. Observou-se que as PVHIV que tiveram frequência mínima de $1 \%$ até 
$75 \%$ sobreviveram em média de 12 anos a 13,1 anos, enquanto aqueles que nunca realizaram tratamento a sobrevida foi significativamente menor, sendo de apenas 4,7 anos. Porém, dado que a chance de um indivíduo diagnosticado nos últimos anos da pesquisa ser assíduo, a sobrevida média do grupo com assiduidade entre 76 e 100\% (média de 4,9) foi menor do que a assiduidade dos grupos intermediários.

Esses achados foram semelhantes aos encontrados por Mirzaei et al. (2016) ao estudar uma coorte de 28.873 PVHIV no período de 1987 a 2016, descobriu que a sobrevida média desde o início da AIDS, foi de 13 anos para as PVHIV em uso de TARV, porém para aqueles que não utilizavam, a expectativa foi muito mais baixa, sendo de apenas 3 anos. Cobre et al.(2020), embora em um período menor de acompanhamento (60 meses), encontrou uma média de sobrevida 54,8 e 49,7 meses para as mulheres e homens, respectivamente, quando em uso regular de TARV.

Nesse sentido, Poorolajal et al. (2016) através de sua pesquisa de revisão com 57 estudos com 294.662 participantes, enfatiza que a maioria das pessoas diagnosticadas com HIV, irá progredir para AIDS nos primeiros 10 anos após do diagnóstico, entretanto, se estas receberem a TARV, sobreviverão em média por mais uma década, porém aqueles que não receberem o tratamento, irão morrer dentro de 2 anos após o início da AIDS. Essa situação foi observada pelo Comitê de Mortalidade por AIDS da cidade de Porto Alegre, que ao avaliar 52 casos, constatou que 28 deles, ocorreram em até dois anos do diagnóstico, em decorrência de um grande número de fatores, dentre os quais se destacou a dificuldade na retenção da pessoa no tratamento (Mocellin et al., 2020).

É notório que a assiduidade no tratamento é um dos fatores chaves para a garantia de uma maior sobrevida. Contudo, nesta pesquisa foi demonstrado que mesmo aquele com o mínimo de contato possível com o SAE, conseguiu experimentar uma sobrevida de vida considerável, quando comparado com aqueles apresentavam maiores frequências. Cabe ressaltar que a assiduidade no tratamento não consiste apenas em realizar a retiradas programadas da TARV. Faz-se necessário também manter uma determinada rotina para as avaliações interdisciplinares, realização de exames, minimização de risco a doenças oportunistas, adoção de hábitos de vida mais saudáveis e seguros.

Diante disso, o diagnostico tardio ainda se configura como um dos grandes problemas enfrentados pela equipe do $\mathrm{SAE}$, pois muitas pessoas acabam por descobrir que viviam/vivem com HIV, quando submetidos a internamentos por comprometimento grave no seu estado de saúde, como no caso das doenças/agravos neurológicos. Estes eventos, acrescido ainda a baixa ou ausência de assiduidade tem grande potencial limitador na sobrevida da PVHIV, devido as altas possibilidades de infecções oportunistas devido a falta do tratamento e/ou acompanhamento em saúde.

Reconhece-se que o início precoce da terapia antirretroviral reduz a morbimortalidade em pessoas vivendo com HIV e prolonga a sobrevida. Os benefícios do início precoce e ampliação da cobertura com TARV, podem ser observados na pesquisa de Montaner et al. (2017), na qual os pesquisadores relatam que essa medida esteve fortemente e estaticamente associada a diminuição de novos diagnósticos e incidência do HIV na população, diminuição dos casos de AIDS, aumento na supressão viral nas PVHIV e ainda com estimam que a cada 1\% de PVHIV suprimidos virologicamente pelo uso da HAART, obtêm-se redução de $2 \%$ na morbimortalidade por HIV/AIDS e 1\% na incidência do HIV.

Outros estudos demonstram o notável o impacto que a TARV traz quando iniciada imediatamente após o diagnóstico, como a redução nas taxas de mortalidade, aumento da supressão viral, recuperação da contagem de LT-CD4, redução do risco de transmissão (Boyd et al., 2019; Coffey et al., 2019).

Nos dados encontrados através da pesquisa realizada no SAE, não foi possível visualizar uma diferença significativa na sobrevida para aquelas PVHIV diagnosticadas antes e após 2013. A hipótese para explicar a ausência de destaque, seja ele positivo ou negativo na sobrevida das PVHIV, está baseada no pouco tempo de acompanhamento ao qual cada grupo foi submetido (5,5 anos). 
Outra hipótese que poderia explicar a pouca diferença nas sobrevidas a partir do ano de 2013 quando houve a implantação do tratamento para todos, foi o atraso em iniciar a TARV para os pacientes assintomáticos ou que estivessem com contagem de LT-CD4 acima de $350 \mathrm{cel} / \mathrm{mm}^{3}$. Isso ocorreu devido ao fato de que estes não mantinham regularidade no serviço, só comparecendo para avaliações anuais ou quando apresentavam algum sintoma. Assim, o início da TARV para este público foi ocorrendo gradualmente ao longo dos anos, visto que não há relato no SAE da realização de busca ativa de assintomáticos, evento que de forma indireta pode ter influenciado a sobrevida, especialmente naqueles com diagnóstico antes de 2013.

\section{Considerações Finais}

Pesquisas com o objetivo em se estudar a sobrevida de PVHIV são de fundamental importância, pois possibilitam monitorar os desiquilíbrios na assistência em saúde, tratamento e cuidados, subsidiando ainda o reconhecimento de indicadores que favoreçam a construção de intervenções específicas, os quais por sua vez resultariam em uma melhor qualidade e expectativa de vida.

Diante dos resultados, é admissível reconhecer que o ingresso oportuno da terapia antirretroviral tenha contribuído positivamente na redução da mortalidade e no aumento significativo da sobrevida em pessoas vivendo com HIV. Porém, devido a complexidade envolvida na adesão a TARV, não foi possível reconhecer neste estudo os determinantes que influenciam de forma negativa a não utilização do tratamento, evento que passa a assumir uma direta correlação a sobrevida destas pessoas.

Torna-se importante então, avaliar a sobrevida de PVHIV sobre os mais diversos contextos, assumindo que a AIDS conseguiu ultrapassar com êxito todos os modelos biomédicos pré-concebidos, seguimentos populacionais e territórios. Realizar o estudo da sobrevida neste público requer a compreensão que esta doença faz interface com diversos condicionantes sociais, como fatores psicológicos, econômicos, culturais, étnico/raciais, além da idade, gênero, escolarização, regionalização e acima de tudo sofre ainda influência da experiência individual de cada pessoa. Talvez se estes não forem considerados na integralidade, não seja possível tentar explicar a menor sobrevivência apresentada em PVHIV, quando avaliadas em variáveis específicas.

\section{Referências}

Akbari, M., Fararouei, M., Haghdoost, A. A., Gouya, M. M., \& Kazerooni, P. A. (2019). Survival and associated factors among people living with HIV/AIDS: A 30-year national survey in Iran. Journal of Research in Medical Sciences: The Official Journal of Isfahan University of Medical Sciences, 24(1), 5. https://doi.org/10.4103/jrms.JRMS_630_18

Alexander, K. A. (2020). Social determinants of HIV/AIDS and intimate partner violence: interrogating the role of race, ethnicity and skin color. Revista Latino-Americana de Enfermagem, 28, e3280. https://doi.org/10.1590/1518-8345.0000.3280

Betancur, M. N., Lins, L., Oliveira, I. R. D., \& Brites, C. (2017). Quality of life, anxiety and depression in patients with HIV/AIDS who present poor adherence to antiretroviral therapy: a cross-sectional study in Salvador, Brazil. Brazilian Journal of Infectious Diseases, 21(5), 507-514. http://dx.doi.org/10.1016/j.bjid.2017.04.004

Boyd, M. A., Boffito, M., Castagna, A., \& Estrada, V. (2019). Rapid initiation of antiretroviral therapy at HIV diagnosis: definition, process, knowledge gaps. HIV medicine, 20(S1), 3-11. https://doi.org/10.1111/hiv.12708

Cerqueira, M. B. R., \& Rodrigues, R. N. (2016). Fatores associados à vulnerabilidade de idosos vivendo com HIV/AIDS em Belo Horizonte (MG), Brasil. Ciência \& Saúde Coletiva, 21(11), 3331-3338. https://doi.org/10.1590/1413-812320152111.14472015

Chaves, R. B. (2018). Óbitos Associados Ao HIV/AIDS De Pessoas Acompanhadas em Serviço Especializado: uma análise de sobrevivência. [Dissertação de Mestrado, Unversidade Federal da Paraíba].

Cobre, A. F., Pedro, C. D. A. A., Fachi, M. M., Vilhena, R. O., Marson, B. M., Nicobue, V., Tonin, F.S., \& Pontarolo, R. (2020). Five-year survival analysis and predictors of death in HIV-positive serology patients attending the Military Hospital of Nampula, Mozambique. AIDS care, 1-9. https://doi.org/10.1080/09540121.2020.1761938

Coffey, S., Bacchetti, P., Sachdev, D., Bacon, O., Jones, D., Ospina-Norvell, C., Torres, S., Lynch, E., Camp, C., Mercer-Slomoff, R., Lee, S., Christopoulos, K., Pilcher, C., Hsu, L., Jin, C., Scheer, S., Havlir, D., \& Gandhi, M. (2019). RAPID antiretroviral therapy: high virologic suppression rates with immediate 
antiretroviral therapy initiation in a vulnerable urban clinic population. AIDS (London, England), 33(5), 825-832. https://doi.org/10.1097/QAD.0000000000002124

de Coninck, Z., Hussain-Alkhateeb, L., Bratt, G., Ekström, A. M., Gisslén, M., Petzold, M., \& Svedhem, V. (2018). Non-AIDS mortality is higher among successfully treated people living with HIV compared with matched HIV-negative control persons: A 15-year follow-up cohort study in Sweden. AIDS Patient Care and STDs, 32(8), 297-305. https://doi.org/10.1089/apc.2018.0015

Costa, A. R., Nobre, C. M. G., Gomes, G. C., Ribeiro, J. P., Mota, M. S., Minasi, A. S. A. (2020). Mudanças no viver a partir do diagnóstico da criança/adolescente com HIV/AIDS e facilidades encontradas no cuidado. Research, Society and Development, 9(7), e55973707. https://doi.org/10.33448/rsdv9i7.3707

Earnshaw, V. A., Bogart, L. M., Dovidio, J. F., \& Williams, D. R. (2015). Stigma and racial/ethnic HIV disparities: Moving toward resilience. Stigma and Health, 1(S), 60-74. https://doi.org/10.1037/2376-6972.1.S.60

Fialho, C. X., Prates, L. A., Oliveira, G., Gomes, N. da S., Escobal, A. P. de L., Cardoso, L. S. (2020). A atuação do enfermeiro frente à gestante vivendo com HIV/Aids. Research, Society and Development, 9(7), e892974545. https://doi.org/10.33448/rsd-v9i7.4545

Figueirêdo Júnior, E. C. F., Ribeiro, A. D., de Araújo Cruz, J. H., Marques, M. H. V. P., Marinho, S. A., \& Pereira, J. V. (2020). Perfil epidemiológico dos casos de Aids notificados no Brasil entre os anos de 2009 a 2019. Research, Society and Development,9(9), e302997233-e302997233. https://doi.org/10.33448/rsd-v9i9.7233

Glynn, J. R., Sonnenberg, P., Nelson, G., Bester, A., Shearer, S., \& Murray, J. (2007). Survival from HIV-1 seroconversion in Southern Africa: a retrospective cohort study in nearly 2000 gold-miners over 10 years of follow-up. Aids, 21(5), 625-632. https://doi.org/10.1097/QAD.0b013e328017f857

Haddad, N., Robert, A., Weeks, A., Popovic, N., Siu, W., \& Archibald, C. (2019). HIV: HIV in Canada-Surveillance Report, 2018. Canada Communicable Disease Report, 45(12), 304-312. https://doi.org/10.14745/ccdr.v45i12a01

IBM Corp. (2012). Released 2012. IBM SPSS Statistics for Windows, Version 21.0. Armonk, NY: IBM Corp.

Jaffar, S., Grant, A. D., Whitworth, J., Smith, P. G., \& Whittle, H. (2004). The natural history of HIV-1 and HIV-2 infections in adults in Africa: a literature review. Bulletin of the World Health Organization, 82, 462-469.

Knauth, D. R., Hentges, B., Macedo, J. L. D., Pilecco, F. B., Teixeira, L. B., \& Leal, A. F. (2020). O diagnóstico do HIV/aids em homens heterossexuais: a surpresa permanece mesmo após mais de 30 anos de epidemia. Cadernos de Saúde Pública, 36(6), e00170118. https://doi.org/10.1590/0102-311X00170118

Lavreys, L., Baeten, J. M., Chohan, V., McClelland, R. S., Hassan, W. M., Richardson, B. A., Mandaliya, K., Achola, J.O.N., \& Overbaugh, J. (2006). Higher set point plasma viral load and more-severe acute HIV type 1 (HIV-1) illness predict mortality among high-risk HIV-1-infected African women. Clinical Infectious Diseases, 42(9), 1333-1339. https://doi.org/10.1086/503258

Lima, T. D. A., Beyrer, C., Golub, J. E., Mota, J. C. D., Malta, M. S., Silva, C. M. F. P. D., \& Bastos, F. I. (2018). Inequalities in HAART uptake and differential survival according to exposure category in Rio de Janeiro, Brazil. Cadernos de Saúde Pública, 34(8), e00009617. https://doi.org/10.1590/0102$311 \mathrm{X} 00009617$

Loch, A. P., Caraciolo, J. M. D. M., Rocha, S. Q., Fonsi, M., Souza, R. D. A., Gianna, M. C., Gonçalves, A. \& Kalichman, A. O. (2020). Intervenção para a implementação do monitoramento clínico em serviços especializados de atenção às pessoas vivendo com HIV/aids. Cadernos de Saúde Pública, 36(5). https://doi.org/10.1590/0102-311x00136219

Mangal, T. D., Meireles, M. V., Pascom, A. R. P., de Almeida Coelho, R., Benzaken, A. S., \& Hallett, T. B. (2019). Determinants of survival of people living with HIV/AIDS on antiretroviral therapy in Brazil 2006-2015. BMC infectious diseases, 19(1), 206. https://doi.org/10.1186/s12879-019-3844-3

May, M. T., Gompels, M., Delpech, V., Porter, K., Orkin, C., Kegg, S., Hay, P., Johnson, M., Palfreeman, A., Gilson, R., Chad wick, D., Martin, F., Hill, T., Walsh, J., Post, F., Fisher, M., Ainsworth, J., Jose, S., Leen, C., Nelson, M., Anderson, J., Sabin, C., UK Collaborative HIV Cohort (UK CHIC) Study. (2014). Impact on life expectancy of HIV-1 positive individuals of CD4+ cell count and viral load response to antiretroviral therapy. AIDS (London, England), 28(8), 1193-1202. https://doi.org/10.1097/QAD.0000000000000243

Melo, M. C., Donalisio, M. R., \& Cordeiro, R. C. (2017). Sobrevida de pacientes com AIDS e coinfecção pelo bacilo da tuberculose nas regiões Sul e Sudeste do Brasil. Ciência \& Saúde Coletiva, 22(11), 3781-3792. https://doi.org/10.1590/1413-812320172211.26352015

Melo, M., Mesquita, F., Barros, M., La-Rotta, E., \& Donalisio, M. (2019). Sobrevida de pacientes com aids e associação com escolaridade e raça/cor da pele no Sul e Sudeste do Brasil: estudo de coorte, 1998-1999. Epidemiologia e Serviços de Saúde. 2019, 28(1), e2018047. https://doi.org/10.5123/S167949742019000100012

Ministério da Saúde. (2013). Protocolo Clínico e Diretrizes Terapêuticas para Manejo da Infecção pelo HIV em Adultos.

Ministério da Saúde. (2018). Protocolo Clínico e Diretrizes Terapêuticas para Manejo da Infecção pelo HIV em Adultos.

Ministério da Saúde (2019). Protocolo Clínico e Diretrizes Terapêuticas para Prevenção da Transmissão Vertical do HIV, Sífilis e Hepatites Virais.

Ministério da Saúde. (2020). Boletim Epidemiológico (p. 68). Brasília: Departamento de Doenças de Condições Crônicas e Infecções Sexualmente Transmissíveis, Secretaria de Vigilância em Saúde.

Mirzaei, M., Farhadian, M., Poorolajal, J., Kazerooni, P. A., Tayeri, K., \& Mohammadi, Y. (2019). Survival rate and the determinants of progression from HIV to AIDS and from AIDS to the death in Iran: 1987 to 2016. Asian Pacific Journal of Tropical Medicine, 12(2), 72. https://doi.org/10.4103/19957645.250840

Miyada, S., Garbin, A. J. Í, Gatto, R. C. J., \& Garbin, C. A. S. (2017). Treatment adherence in patients living with HIV/AIDS assisted at a specialized facility in Brazil. Revista da Sociedade Brasileira de Medicina Tropical, 50(5), 607-612. http://dx.doi.org/10.1590/0037-8682-0266-2017 
Mocellin, L. P., Winkler, G. B., Stella, I. M., Vieira, P. C., Beck, C., Behar, P. R. P., \& Kuchenbecker, R. D. S. (2020). Caracterização dos óbitos e dos itinerários terapêuticos investigados pelo Comitê Municipal de Mortalidade por Aids de Porto Alegre em 2015. Epidemiologia e Serviços de Saúde, 29, 29(3), e2019355. https://doi.org/10.5123/S1679-49742020000300009

Montaner, J. S., Lima, V. D., Harrigan, P. R., Lourenço, L., Yip, B., Nosyk, B., Wood, E., Kerr, T., Shannon, K., Moore, D., Hogg, R.S., Barrios, R., Gilbert, M., Krajden, M., Gustafson, R., Daly, P., \& Kendall, P. (2014). Expansion of HAART Coverage Is Associated with Sustained Decreases in HIV/AIDS Morbidity, Mortality and HIV Transmission: The "HIV Treatment as Prevention" Experience in a Canadian Setting. PLoS one, 9(2), e87872. https://doi.org/10.1371/journal.pone.0087872

Morgan, D., Mahe, C., Mayanja, B., Okongo, J. M., Lubega, R., \& Whitworth, J. A. (2002). HIV-1 infection in rural Africa: is there a difference in median time to AIDS and survival compared with that in industrialized countries? Aids, 16(4), 597-603.

Müller, E. V., \& Borges, P. K.O. (2020). Sobrevida de pacientes HIV/AIDS em tratamento antirretroviral e fatores associados na região dos Campos Gerais, Paraná: 2002-2014/HIV/AIDS patients survival on antiretroviral treatment and associated factors in the Campos Gerais region, Paraná state: 20022014. Brazilian Journal of Development, 6(5), 28523-28542. https://doi.org/10.34117/bjdv6n5-339

Oliveira, F. B. M., Moura, M. E. B., de Araújo, T. M. E., \& Andrade, E. M. L. R. (2015). Qualidade de vida e fatores associados em pessoas vivendo com HIV/AIDS. Acta Paulista de Enfermagem, 28(6), 510-516. https://doi.org/10.1590/1982-0194201500086

Palumbo, R. (2015). Discussing the effects of poor health literacy on patients facing HIV: a narrative literature review. International journal of health policy and management, 4(7), 417-430. https://doi.org/10.15171/ijhpm.2015.95

Pereira, G., Shimizu, H., Bermudez, X., \& Hamann, E. (2018). Epidemiologia do HIV e aids no estado do Rio Grande do Sul, 1980-2015. Epidemiologia E Serviços De Saúde, 27(4), e2017374. https://doi.org/10.5123/s1679-49742018000400004

Poorolajal, J., Hooshmand, E., Mahjub, H., Esmailnasab, N., \& Jenabi, E. (2016). Survival rate of AIDS disease and mortality in HIV-infected patients: a meta-analysis. Public Health, 139, 3-12. https://doi.org/10.1016/j.puhe.2016.05.004

Rossi, S. M. G. D., Maluf, E. C. P., Carvalho, D. S., Ribeiro, C. E. L., \& Battaglin, C. R. P. (2012). Impacto da terapia antirretroviral conforme diferentes consensos de tratamento da Aids no Brasil. Revista Panamericana de Salud Pública, 32(2), 117-123.

Tancredi, M. V., \& Waldman, E. A. (2014). Survival of AIDS patients in Sao Paulo-Brazil in the pre-and post-HAART eras: a cohort study. BMC Infectious Diseases, 14(1), 1-8. https://doi.org/10.1186/s12879-014-0599-8

Trickey A., May, M. T., Vehreschild, J. J., Obel, N., Gill, M. J., Crane, H. M., Boesecke, C., Patterson, S., Grabar, S., Cazanave, C., Cavassini, M., Shepherd, L., Monforte, A.A., Sighem, A. V., Saag, M., Lampe, F., Hernando, V., Montero, M., Zangerle, Z., Justice, A.C., Sterling, T., Ingle, S. M., Sterne, J. A. C. (2017). Survival of HIV-positive patients starting antiretroviral therapy between 1996 and 2013: a collaborative analysis of cohort studies. The lancet HIV. 2017, 4(8), e349-e356. https://doi.org/10.1016/S2352-3018(17)30066-8

Velame, K. T., Silva, R. D. S. D., \& Cerutti Junior, C. (2020). Factors related to adherence to antiretroviral treatment in a specialized care facility. Revista da Associação Médica Brasileira, 66(3), 290-295. https://doi.org/10.1590/1806-9282.66.3.290

Vieira, G.D, dos Reis, A. R. P., de Alcântara Augusto, F. O. T., Martins, K. R., Kern, P. R. F., de Souza, T. F., Camargo, S.A \& de Sousa, C. M. (2015). Characteristics relating to the interiorization of acquired immunodeficiency syndrome in Brazil: a cross-sectional study. Infectious diseases of poverty, 4(1), 31. https://doi.org/10.1186/s40249-015-0060-2

Word Health Organization. (2016). Consolidated guidelines on the use of antiretroviral drugs for treating and preventing HIV infection: recommendations for a public health approach. World Health Organization.

Zeng, Y. L., Tang, H. L., Li, J. M., Wang, Q. S., Yu, H., Su, L., Yang, W., Gong, Y., Li, T., Huang, W.L., Zhang, L. L., \& Lai, W. W. (2019). Survival analysis of people living with HIV/AIDS in Sichuan province, 1991-2017. Zhonghua liu xing bing xue za zhi= Zhonghua liuxingbingxue zazhi, 40(3), 309314. https://doi.org/10.3760/cma.j.issn.0254-6450.2019.03.010

Zhang, G., Gong, Y., Wang, Q., Deng, L., Zhang, S., Liao, Q., Yu, G., Wang, J., Ye, S., \& Liu, Z. (2016). Outcomes and factors associated with survival of patients with HIV/AIDS initiating antiretroviral treatment in Liangshan Prefecture, southwest of China: A retrospective cohort study from 2005 to 2013.

Medicine, 95(27), e3969. https://doi.org/10.1097/MD.000 